Gefässchirurgie 2018 $23: 564-565$ https://doi.org/10.1007/s00772-018-0484-7

(C) Springer Medizin Verlag GmbH, ein Teil von Springer Nature 2018

CrossMark

\section{A. Larena-Avellaneda}

Klinik und Poliklinik für Gefäßmedizin, Gefäßchirurgie - Endovaskuläre Therapie - Angiologie, Universitäres Herzzentrum Hamburg, Universitätsklinikum Hamburg-Eppendorf, Hamburg, Deutschland

\title{
Stille Nacht - Plädoyer für mehr Achtsamkeit
}

Geschätzte Leserinnen und Leser,

schon wieder ist ein Jahr fast vorbei - zumindest, wenn Sie diese Ausgabe in den Händen halten. Das Editorial für Heft 8 schreibe ich am liebsten: Rückblick auf das Jahr, Gedanken für die Zukunft - und es ist wieder bald Weihnachten. Letztes Jahr hatte ich an gleicher Stelle unter dem Titel „Alle Jahre wieder" um vermehrte Einsendungen von Artikeln gebeten und auch nochmals das Thema „Journal Impact Factor" (JIF) adressiert. Ich will Sie damit nicht langweilen. Aber dennoch möchte ich Sie auf dem Laufenden halten, da wir auf der Herausgebersitzung im Oktober in Bonn die aktuellen Zahlen vorstellten. Was man festhalten kann: Die Open Access publizierten Artikel (Open Choice) werden deutlich häufiger zitiert als die anderen. Und Zitationen sind wesentliche Grundvoraussetzung für den JIF. Mithilfe des Vorstandes der DGG und der Autoren wurde nun eine englische Übersetzung der deutschen Kurzfassung der deutschen Leitlinien zum abdominellen Aortenaneurysma (diese wurde Ihnen in Heft 6 präsentiert) online gestellt [1]. Die Übersetzung der S2K-Leitlinie Aortendissektionen (Kurzfassung publiziert im letzten Heft) wird folgen. Hierdurch soll - analog zur Europäischen Gesellschaft und deren Leitlinienpublikationen im European Journal of Vascular and Endovascular Surgery - die Anzahl der Zitationen deutlich gesteigert werden. Insofern blicken wir optimistisch in die Zukunft und hoffen darauf, die Voraussetzungen für den JIF in 2021 erfüllen zu können.

Apropos Optimismus: Derzeit höre ich ein sehr beunruhigendes Buch: Den Bestseller 21 Lektionen für das 21. Jahr- hundert von Yuval Harari [2]. Der Autor gilt als einer der bedeutendsten Denker und seine Theorien zur Macht der Algorithmen und Verschmelzung von Informations- und Biotechnologie sind erschreckend - auf fast allen Ebenen. Auch im Arztberuf wird in wenigen Jahrzehnten wenig so sein wie heute, und viele unserer Aufgaben werden von Computern/ Netzwerken/Algorithmen übernommen worden sein. In seinen Ausführungen zu (künstlicher) Intelligenz und Bewusstsein zitiert er dabei Tristan Harris, einen ehemaligen Manager von Google, und seine Ideen zu „Time Well Spent“. Es geht hier insbesondere auch um Ethik in der Technologie [3]. Die zentrale These lautet: „Our society is being hijacked by technology. What began as a race to monetize our attention is now eroding the pillars of our society: mental health, democracy, social relationships, and our children." Weiterhin wird ausführlich beschrieben, mit welchen Tricks sich die Social-MediaNetzwerke die Online-Zeit ihrer Nutzer verlängern. Als Folgen hiervon werden Depression, Vereinsamung und auch eine erhöhte Selbstmordrate befürchtet [4].

Warum erzähle ich das hier? Weil wir es genauso machen und auch täglich mitbekommen. Schauen Sie sich mal in einem Meeting um. Mindestens 2-3, oft hoch dekorierte Ärzte, Manager oder Geschäftsführer haben ihr Smartphone in der Hand und erledigen einen Teil der inzwischen unmenschlichen E-Mail-Flut. Dennoch - mit den Gedanken sind diese Leute woanders. Haben wir nicht mehr die Zeit, uns 45 min auf unser Gegenüber zu konzentrieren? Oder sind die Inhalte so langweilig (warum sind wir dann überhaupt in diesem Meeting?)?
Gehen Sie mal über einen Campus, oder egal wo: ca. $50-80 \%$ der jüngeren Passanten reden mit ihrem Handy oder schauen darauf. Man ist zwar physisch anwesend, aber nicht geistig. Und auch hier ertappe ich mich selbst: Bisweilen habe ich den Fahrstuhl verpasst, weil ich mit dem Smartphone beschäftigt war. Die Beobachtung gilt auch für Vorlesungen: Eine große Zahl an Studenten hat ihren PC mitgebracht und aufgeklappt oder ein Tablet oder eben ein Smartphone vor sich liegen. Im besten Fall werden meine Ausführungen zeitgleich dokumentiert, im zweitbesten Fall die Vorlesung heruntergeladen oder eben etwas anderes getan. Zum einen empfinde ich dies als Missachtung des Dozenten (ebenso wie essen im Hörsaal während der Vorlesung), zum anderen bezweifle ich sehr, dass das Wissen auch nur ansatzweise hängenbleibt, wenn man nicht mitdenkt. Meines Erachtens ist das alles mangelnde Fokussierung oder mangelnde Achtsamkeit.

Aber es sind nicht nur Smartphones, die uns die Achtsamkeit nehmen. Auch der Zeitmangel ist hier kein guter Ratgeber, wie ich zuletzt selbst mal wieder gemerkt habe. Aufgrund von Krankheitsfällen hatte ich mehrere Aufgaben in der Klinik parallel übernommen. So kam dann ein Anruf von unserer zentralen Notaufnahme (ZNA), ein Patient mit Verdacht auf akuten arteriellen Verschluss sei eingewiesen worden. Ich hielt es für eine gute Idee, um Zeit zu sparen, den Patienten in unsere Poliklinik bringen zu lassen. Diese ist ein Stück von der ZNA im Hauptgebäude entfernt (5 min für einen Fußgänger ohne pAVK) und Patienten benötigen einen Transport. Nach $2 \mathrm{~h}$ erschien der Patient in unserer Poliklinik, und unser Dienstarzt fragte mich, 
ob ich den Patienten gesehen hätte. Tatsächlich hatte der Patient Fußpulse. Hätte ich mir die 15 min genommen, den Patienten in der Notaufnahme aufzusuchen und zu untersuchen, hätte man viel Zeit und Ressourcen sparen können. Ist es schon so weit, dass man keine $15 \mathrm{~min}$ mehr hat? An diesem Tag hatte ich den Eindruck, diese $15 \mathrm{~min}$ sparen zu können. Tippen wir nicht alle auf unseren Dienst-Smartphones herum, um E-Mails zu erledigen, und hören der Umwelt nicht $\mathrm{zu}$ ?

Und damit komme ich wieder zur Achtsamkeit und warum der Beruf des Chirurgen hier etwas Besonderes ist. Wie schon in einem früheren Editorial beschrieben, ist die Operation selbst etwas Einzigartiges und geht mit Glücksgefühlen (Flow) einher [5]. Vor allen Dingen: Wir haben hier KEIN Handy, auf das wir dauernd schauen (auch wenn wir es bisweilen mit in den OP nehmen und es vielleicht klingelt). Wir MÜSSEN für diese Zeit extrem achtsam sein, sonst wird die Operation schiefgehen. Also, es geht doch!

Dies wiederum leitet wunderbar zur Einführung zum Thema der vorliegenden Ausgabe hin: Perioperative Medizin. Die Achtsamkeit, die wir beim Operieren an den Tag legen, darf mit Verlassen des Operationssaals nicht abgelegt werden. Der Patient sollte regelmäßig vom Operateur visitiert werden, damit dieser sein Ergebnis kennt - und daraus für sich selbst lernt. Ein altes Zitat, dessen Urheber mir leider entfallen ist, lautet:

Die Operation ist keine Trophäe für den Chirurgen. Sie dient dem Wohl des Patienten.

Und genauso wichtig wie die Operation selbst, sind auch die perioperativen Maßnahmen. Gerade die perioperative Medizin in den Händen von Chirurgen sorgt dafür, dass Chirurgen eben nicht nur Handwerker und Erfüllungsgehilfen sind. Tatsächlich ist die Bedeutung der perioperativen Medizin und Ethik auch medizinhistorisch wichtig, da Chirurgen ja, zusammen mit den Barbieren, von der Ärzteschaft lange ausgeschlossen waren [6]. Dabei spielen zahlreiche Aspekte eine wichtige Rolle, von denen hier nur einige wenige abgedeckt werden (können). Auch dies zeigt meines Erachtens schöne Besonderheiten unseres Fachs: Die Diagnostik und Indikationsstellung liegen weitestgehend in den Händen des Gefäßmediziners. Dies spiegelt insbesondere der Beitrag von W. Schierling (Ultraschall) wider. Gefäßchirurgen sind heutzutage aber auch interventionell und damit radiologisch tätig. Auf diesem Gebiet tut sich derzeit unheimlich viel, um die Strahlenbelastung von Patienten und Therapeuten $\mathrm{zu}$ reduzieren. M. Kaschwich aus der Arbeitsgruppe von M. Kleemann beschreibt in seinem Artikel „Möglichkeiten zur Strahlenreduktion im OP“. Ein weiterer Weg, Strahlen zu reduzieren, ist die Qualitätskontrolle unter Verzicht auf eine Angiografie. Neben dem Ultraschall ist hier insbesondere die Flussmessung („transit time flow measurement" - TTFM) zu nennen. Während dies für die Herzchirurgie weitestgehend standardisiert ist, gibt es für gefäßchirurgische Anwendungen nur wenig Literatur. Ich konnte Pirkka Vikatmaa aus Finnland für einen Übersichtsartikel und die Darstellung seiner eigenen Erfahrungen gewinnen.

$\mathrm{Zu}$ den Pflichten eines Chirurgen gehört im Rahmen der regelmäßigen Visite auch die Schmerztherapie - prä- UND postoperativ. H. Wenk von der Kommission „Perioperative Medizin“ hat hierzu eine aktuelle Übersicht verfasst. Zwar nicht zum Leitthema eingeladen, aber genau passend hat $R$. Grundmann für die Rubrik „Originalien“ ein Manuskript zur Ernährung bei pAVK eingereicht - ein Thema, dass in meinen Augen von uns viel zu wenig Beachtung findet. Wie Sie also sehen, werden verschiedene Gebiete gestreift.

Weiterhin finden Sie in der vorliegenden Ausgabe einen interessanten Artikel zur Wirkung von Chirurgen in der anatomischen Lehre (A. Boeckers) und einen interessanten Case Report (C. Schmaus). In der heutigen Ausgabe geht es bei der CME-Fortbildung um die Chimney-Technik (K. P. Donas), und F. Simon berichtet über den Aufbau von Biobanken.

Ich wünsche Ihnen also viel Achtsamkeit und Spaß mit der vorliegenden
Ausgabe der Gefässchirurgie und eine gesegnete, beschauliche Weihnachtszeit.

Herzlichst, Ihr

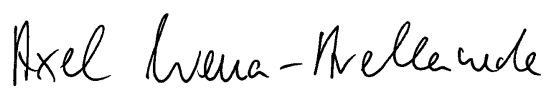

Prof. Dr. A. Larena-Avellaneda

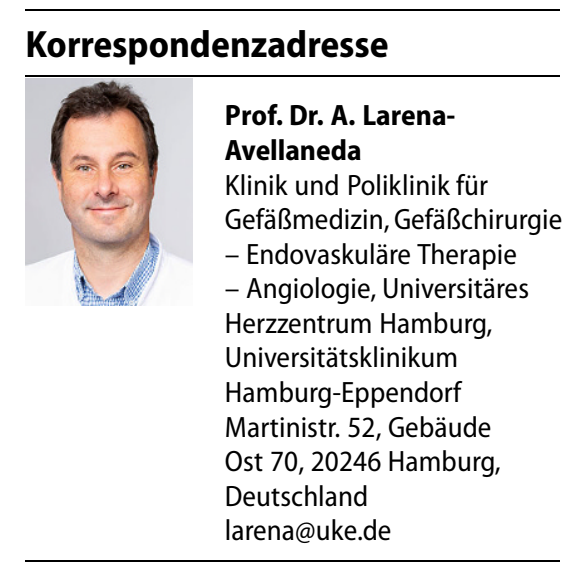

Interessenkonflikt. A. Larena-Avellaneda gibt an, dass kein Interessenkonflikt besteht.

\section{Literatur}

1. Debus ES, Heidemann F, Gross-Fengels W et al (2018) Gefasschirurgie. https://doi.org/10.1007/ s00772-018-0465-x

2. Harari YN (2018) (2018) 21 Lektionen für, 1. Aufl. das, Bd. 21. Beck Verlag, Jahrhundert. CH

3. http://humanetech.com/problem/

4. Stierler W (2018) Die Rattenfänger aus dem Silicon Valley. Technol Rev 2:30

5. Larena-Avellaneda A (2018) Früher war alles besser? (Tempora mutantur pars II). Gefasschirurgie 4:18

6. Namm JP, Siegler M, Brander C, Kim TY, Lowe C, Angelos P (2014) History and Evolution of Surgical Ethics: John Gregory to the Twenty-first Century. World J Surg 38:1568-1573 